OPINIONS

\title{
We Cannot Allow a Wikipedia Gap!
}

\author{
Sage Ross*
}

Editing Wikipedia is probably the best way for historians of science to spend their working hours. I became an historian of science because the history (and philosophy and sociology) of science (and medicine and technology) touches on virtually every important social and political issue in the modern world. I wanted to help put the field where it ought to be, at the center of any program of liberal and/or scientific education-part of the baseline of cultural literacy. When I entered grad school, it was a great letdown to realize just how small a part of academic life involves reaching beyond the walls of academe. Public service is not part of the scholar social role until one reaches full professor, if ever. But while my original motivation to edit Wikipedia was an impulse toward service (along with the desire for an audience), that is not primarily why it is incumbent upon scholars in the history of science and her sister fields to join me.

Wikipedia is only beginning to realize its potential for embiggening the role of humanistic knowledge and bridging the ever-widening gaps between the islands of specialized knowledge. Countless surfers have experienced the problem with Wikipedia; an afternoon's intellectual journey that begins with Canada may end with the history of abortion (via "Canadian Charter of Rights and Freedoms" and "Abortion"), the history of biotechnology (via "17th century" and "Timeline of invention"), the Manhattan Project (via "Economy of Canada" and "Nuclear power in Canada"), or philosophy of science (via "Education in Canada", "Bachelor's degree", and "Philosophy").

A Wikipedia article is a sort of billboard in the marketplace of ideas. Highquality content, based on the best scholarly sources, has the power to increase dramatically the demand for our intellectual goods and services. For too long, the noncommercial culture of the academy has shackled itself to the system of inaccessible journal publications. (Spontaneous Generations, only the second open access history and philosophy of science journal so far as I know, is an overdue step in the right direction.) Our field in particular has plenty of mind share to gain; so much of what we do is highly relevant to, yet under-read by, other humanists. With a stronger Wikipedia presence, garden-variety historians and other scholars will be able to engage with our work more often. History departments may even begin to realize the pressing need for an additional

\footnotetext{
* Sage Ross is a PhD candidate in the History of Science and Medicine Program at Yale University. $\mathrm{He}$ is writing a dissertation on the history of molecular evolution. Inspired by his time at the University of Oklahoma, he plans to get a large tattoo of Kepler's nested solids to show his devotion to the history of science. Sage blogs at Revise and Dissent, a group blog on the History News Network. As 'ragesoss' on Wikipedia, he has written featured articles on Johannes Kepler, History of biology, and Rachel Carson; he is eager for criticism.
}

Spontaneous Generations 1:1 (2007). ISSN 1913-0465. University of Toronto 
historian of science or three. Even on an individual level, making sure your area of research is represented amply and accurately will draw people to your work, especially for those interested in related topics.

Wikipedia also has some unique-and enjoyable-challenges for professional scholars. Rarely do we get a chance to forge an article-length overview of the history of an entire discipline; even full books on such predetermined topics are often left to scientists, despite the wealth of detailed historical studies that could be used to such ends. A coherent and concise treatment of broader subjects like "history of technology," "history of biology" (the one I've been working on lately), and toughest of all, "history of science" may not even be possible. But the demand for such narratives is tremendous, and the attempt would do much toward putting our own sprawling field into perspective... a sort of living bibliography. Writing for a general audience is also an underdeveloped skill among members of our esoteric invisible college.

WikiProject History of Science, an effort to organize and facilitate collaboration on Wikipedia's history of science, medicine and technology content, has grown steadily since it launched in January 2006; there are now over 70 members. However, this includes relatively few with formal training in our field (and for most of them, only undergraduate training). This is a typical problem for humanities fields on Wikipedia, but it is not universal; for example, the Russian History WikiProject, spearheaded by prominent Russianist Marshall Poe, has at least 14 members with related doctorates, and that many more graduates students and other scholars. Other disciplines are similarly ahead of the curve here: Linguist List recently set aside funding for a graduate student to coordinate linguistics editing, while the ever-popular field of military history has about 600 active Wikipedians, working on about 34,000 articles (including over a tenth of Wikipedia's Featured Articles). Getting involved sooner rather than later will help set our work apart and lay the cultural groundwork for long-term disciplinary success.

Incorporating Wikipedia into courses is probably the best way to begin. As I have found over the last two semesters, students respond well to editing assignments when the audience is the wide world of Wikipedia readers. They also come away with a healthy skepticism about what they read on Wikipedia. We should not be teaching our undergraduates how to be mini professional historians, we should be training them to be versatile and savvy media consumers who spot junk when they see it. There are plenty of Wikipedians who will be eager to help manage such assignments, and (as you may have noticed) there is no shortage of articles in need of work.

An encyclopedia that anyone can edit is not without drawbacks. The refrain goes something like this: "Why should I post my magnificent prose just so it can be degraded and destroyed by high school students and independent scholars?"; "What does this anonymous work do for me professionally, when I don't even get a byline?"; "As just another internet pseudonym, how can I overcome the ignorant masses in my controversial area of expertise?". The reality of "anyone 
can edit" is not so bad as it seems: as one editor was quoted in a recent New York Times piece, "The problem with Wikipedia is that it only works in practice. In theory, it can never work." In practice, the good and well-sourced content in most articles is remarkably stable; usually the worst long-term threat to a solid article is the accretion of trivial details. Regarding professional credit, it will be up to us to create the future academy, and we are free to rewrite the definitions of scholarship and service to the profession. As for the heated topics, where myth and enthusiasm abound-there is no easy answer, but these are fights worth fighting. And (at least one page at a time) the myths we dispel on Wikipedia tend to stay dispelled.

SAGE ROSS

Yale University

Recommended reading:

O'Donnell, Daniel Paul. 2007. If I were "You": How academics can stop worrying and learn to love "the encyclopedia that anyone can edit". The Heroic Age, May.

Miller, Christopher. 2007. Strange facts in the history classroom: Or how I learned to stop worrying and love the Wiki(pedia). Perspectives, May.

Willinsky, John. 2007. What open access research can do for Wikipedia. First Monday, March. 